\title{
Entanglement from the Dynamics of an Ideal Bose Gas in a Lattice
}

\author{
Sougato Bose \\ Department of Physics and Astronomy, University College London, Gower St., London WC1E 6BT, UK
}

\begin{abstract}
We show how the remotest sites of a finite lattice can be entangled, with the amount of entanglement exceeding that of a singlet, solely through the dynamics of an ideal Bose gas in a special initial state in the lattice. When additional occupation number measurements are made on the intermediate lattice sites, then the amount of entanglement and the length of the lattice separating the entangled sites can be significantly enhanced. The entanglement generated by this dynamical procedure is found to be higher than that for the ground state of an ideal Bose gas in the same lattice. A second dynamical evolution is shown to verify the existence of these entangled states, as well entangle qubits belonging to well separated quantum registers.
\end{abstract}

PACS numbers:

One of the aims in the field of quantum information is to set up entanglement between locations separated by some distance, and in general, greater the separation and more the amount of this entanglement, the better. While photons are best for long distance entanglement distribution, for short distances (such as for linking quantum registers) other alternatives are important [1]. In this context, the dynamics of spin chains have been proposed for the distribution of entanglement over a distance of several lattice sites (For example, Refs. 2, 3, 44 to mention a very few). However, as the number of possible states of a spin in a spin chain is low, the amount of entanglement that can be dynamically generated and distributed through a single spin chain channel in a limited time is restricted. In this paper, as an alternative to spin chains, we suggest the use of the dynamics of an ideal gas of $M$ bosons in a lattice to generate and distribute entanglement between its remotest sites. Note that our dynamics, when followed by certain occupation number measurements, will create high but "finite" dimensional entangled states (with entanglement $\sim \log _{2} \sqrt{M}+$ Const.) which are qualitatively very different from the infinite dimensional Gaussian entangled states which can be generated dynamically through harmonic oscillator chains [5].

Another motivation for the current work originates from the literature on entangling Bose-Einstein condensates (BECs) of gaseous atoms/molecules in distinct traps (Refs. [6, 7, 8, 9, 10] to mention a very few) where usually small lattices or continuous variable entanglement are considered. Can we use lattice dynamics to create entanglement between traps separated by several intervening lattice sites? Here we show how to accomplish this without either the physical movement of traps or any local modulation of the lattice parameters.

We consider a one dimensional lattice of $N$ sites, where the aim is to establish a significant amount of entanglement between sites 1 and $N$. We choose $N$ to be odd and initially place $M$ bosons in the $\frac{N+1}{2}$ th site of the lattice and keep all the other sites empty. Physically this corresponds to a Fock state $|M\rangle$ on the $\frac{N+1}{2}$ th site of the lattice and a vacuum state in all the other sites. In terms

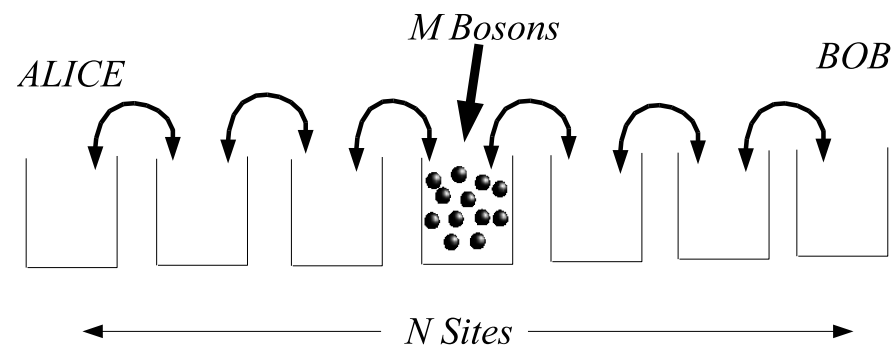

FIG. 1: Our setup of creating entanglement between sites 1 and $N$ of a $1 \mathrm{D}$ lattice. One simply starts with $M$ bosons in the $(N+1) / 2$ th lattice sites and allows dynamical evolution of the system to create entanglement between the sites 1 and $N$. Alice and Bob who have access to the sites 1 and $N$ can use the entanglement for quantum communications of for linking distinct quantum registers.

of boson creation operators $a_{j}^{\dagger}$ which create a boson in the $j$ th lattice site the initial state is thus

$$
|\Psi(0)\rangle=\frac{\left(a_{\frac{N+1}{2}}^{\dagger}\right)^{M}}{\sqrt{M !}}|0\rangle .
$$

This special initial state greatly simplifies the calculations and can be regarded as a generalization of a single spin flip at the midpoint of a spin chain, which has been studied for entanglement generation [4]. We assume the bosons to be essentially non-interacting during the timescale of our scheme i.e., their collection is an ideal Bose gas. Then the Hamiltonian of the system in the lattice is

$$
H=J \sum_{j=1}^{N}\left(a_{j}^{\dagger} a_{j+1}+a_{j} a_{j+1}^{\dagger}\right) .
$$

The state of each boson then evolves independently in the lattice (i.e., each boson evolves as if it was hopping alone in an otherwise empty lattice) as $a_{\frac{N+1}{2}}^{\dagger} \rightarrow \sum_{j=1}^{N} f_{j}(t) a_{j}^{\dagger}$ where $f_{j}(t)$ is the amplitude of the transfer of a single boson from the $\frac{N+1}{2}$ th site to the $j$ th site in time $t$. Thus 
the state of the $M$ boson system at time $t$ is

$$
|\Psi(t)\rangle=\frac{\left(\sum_{j=1}^{N} f_{j}(t) a_{j}^{\dagger}\right)^{M}}{\sqrt{M !}}|0\rangle .
$$

The evolution amplitudes $f_{j}(t)$ are identical to that of a $\mathrm{XY}$ spin chain in the single excitation sector, and is given [3, 11] by

$$
f_{j}(t)=\frac{2}{N+1} \sum_{k=1}^{N}\left\{\sin \frac{\pi k}{2} \sin \frac{\pi k j}{N+1}\right\} e^{i 2 J t \cos \frac{k \pi}{N+1}} .
$$

Eqs.(3) and (4) give the complete time evolution of the $M$ boson state analytically.

Our task is now to calculate how much entanglement exists between sites 1 and $N$ in the state of Eq.(3) as a function of time and find a time at which this entanglement is large. We will thus have to calculate the reduced density matrix of the states of sites 1 and $N$ by tracing out the state of all the other sites. To accomplish that we adopt a strategy from Ref. 13], which evaluates the entanglement between two regions of a BoseEinstein condensate in its ground state in a single trap. The strategy is to define new creation/annihilation operators by combining the operators $a_{j}^{\dagger}$ as $E^{\dagger}=f_{1}(t)\left(a_{1}^{\dagger}+\right.$ $\left.a_{N}^{\dagger}\right) / \sqrt{2\left|f_{1}(t)\right|^{2}}$ and $L^{\dagger}=\sum_{j=2}^{N-1} f_{j}(t) a_{j}^{\dagger} / \sqrt{1-2\left|f_{1}(t)\right|^{2}}$, which are valid bosonic creation operators satisfying $\left[E, E^{\dagger}\right]=1,\left[L, L^{\dagger}\right]=1$. Noting that the symmetry of our problem implies $f_{1}(t)=f_{N}(t)$, we expand the expression of $|\Psi(t)\rangle$ in Eq.(3) in terms of the above operators to get

$$
\begin{aligned}
|\Psi(t)\rangle & =\sum_{r=0}^{M} \sqrt{{ }^{M} C_{r}}\left(\sqrt{2}\left|f_{1}(t)\right|\right)^{r}\left(\sqrt{1-2\left|f_{1}(t)\right|^{2}}\right)^{M-r} \\
& \times\left|\psi_{r}\right\rangle_{1 N}\left|\phi_{r}\right\rangle_{2 \ldots N}
\end{aligned}
$$

where we have substituted $\left|\psi_{r}\right\rangle_{1 N}=\frac{\left(E^{\dagger}\right)^{r}}{\sqrt{r !}}|0\rangle$ and $\left|\phi_{r}\right\rangle_{2 \ldots N}=\frac{\left(L^{\dagger}\right)^{M-r}}{\sqrt{(M-r) !}}|0\rangle$. Noting that the set of states $\left\{\left|\phi_{r}\right\rangle_{2 \ldots N}\right\}$ represents an orthonormal set in the space of states of the sites 2 to $N-1$, we have the the reduced density matrix of sites 1 and $N$ to be

$$
\rho(t)_{1 N}=\sum_{r=0}^{M} P_{r}(t)\left|\psi_{r}\right\rangle\left\langle\left.\psi_{r}\right|_{1 N}\right.
$$

where $P_{r}(t)={ }^{M} C_{r}\left(2\left|f_{1}(t)\right|^{2}\right)^{r}\left(1-2\left|f_{1}(t)\right|^{2}\right)^{M-r}$. We can write $\left|\psi_{r}\right\rangle_{1 N}$ in terms of the occupation numbers as

$$
\left|\psi_{r}\right\rangle_{1 N}=\frac{1}{2^{r / 2}} \sum_{k=0}^{r} \sqrt{{ }^{r} C_{k}}|k\rangle_{1}|r-k\rangle_{N} .
$$

Note that the only time dependence of the state $\rho(t)_{1 N}$ stems from $f_{1}(t)$, maximizing which over a long period of time is a pretty good strategy for obtaining a time $t_{h}$ such that $\rho\left(t_{h}\right)_{1 N}$ is highly entangled. The maximization ensures that the proportion of the state $\left|\psi_{M}\right\rangle_{1 N}$, which has the most entanglement among the set of states $\left\{\left|\psi_{r}\right\rangle_{1 N}\right\}$, is the highest possible in $\rho\left(t_{h}\right)_{1 N}$. Ideally, the lattice dynamics should be frozen at $t_{h}$, say by globally raising the barriers between all wells of the lattice, so that Alice and Bob can utilize $\rho\left(t_{h}\right)_{1 N}$ for quantum communications or linking quantum registers. For the smallest non-trivial lattice $(N=3)$ we know that $f_{1}(t)=1 / \sqrt{2}$ at $t=\pi / 2 J \sqrt{2}$ from the XY model [11]. For this case, the state $\left|\psi_{M}\right\rangle_{13}$ is generated between sites 1 and 3 at $t=\pi / 2 J \sqrt{2}$ whose entanglement can be made to grow without limits by increasing $M$. For this special case, the advantage over spin- $1 / 2$ chains is most evident, where only the case of $M=1$ can be realized (with a single flip in the middle of a chain of three spin- $1 / 2$ systems).

In general (for $N>3$ ), the state $\rho(t)_{1 N}$ is a mixed state of a $(M+1) \times(M+1)$ dimensional system, and the only readily computable measure of its entanglement is the logarithmic negativity $E_{n}$ [12], which bounds the amount of pure state entanglement extractable by local actions from the state $\rho(t)_{1 N}$. It is the standard measure used when high dimensional mixed entangled states arise [5]. The $E_{n}$ of $\rho(t)_{1 N}$ for an appropriately chosen time $t=t_{h}$ (see above) are plotted in Fig [2] as a function of $N$ for different values of $M$. This figure clearly shows that for $N$ as high as 21, one can generate more entanglement than that of a singlet $\left(E_{n}=1\right.$ for a singlet). For such modest lengths, thus one generates more entanglement between the ends of a lattice by using a $M>1$ boson gas than ever possible with a spin- $1 / 2$ chain, which is the $M=1$ case, also plotted in Fig 2] The advantages of increasing $M$ diminish, though, as one increases $N$. For $N=51$, we see that though there is some advantage of high boson number $(M=50)$ over the spin chain $(M=1)$ case, this advantage does not increase by increasing $M$ (for example, the $M=5$ and 50 plots are nearly coincident).

Next, we slightly modify our scheme and after the dynamical evolution till $t_{h}$, we measure the total number of bosons in the intermediate sites (sites 2 to $N-1$ ). With probability $P_{r}$, we will find this number to be $M-r$, and when we do so, we will generate the state $\left|\psi_{r}\right\rangle_{1 N}$ between sites 1 and $N$. Note that $\left|\psi_{r}\right\rangle_{1 N}$ is created between sites 1 and $N$ whenever a "total" of $M-r$ bosons is found in the remaining sites irrespective of the distribution of these $M-r$ bosons among the sites. The amount of entanglement in the pure state $\left|\psi_{r}\right\rangle_{1 N}$ is given by its von Neumann entropy of entanglement $E_{v}\left(\left|\psi_{r}\right\rangle_{1 N}\right)=$ $-\operatorname{Tr}\left(\rho_{1} \log _{2} \rho_{1}\right)$, where $\rho_{1}=\operatorname{Tr}_{N}\left(\left|\psi_{r}\right\rangle\left\langle\left.\psi_{r}\right|_{1 N}\right)\right.$, which equals the quantity of entanglement that can be obtained as singlets (the most useful form, say for their use as a resource for perfect teleportation of qubits from Alice to Bob) from the state by local actions and classical communications alone. Thus the average von Neumann entropy of entanglement $\left\langle E_{v}\right\rangle=\sum_{r} P_{r} E_{v}\left(\left|\psi_{r}\right\rangle_{1 N}\right)$ over all possible measurement outcomes is operationally the most 
useful measure of the entanglement between sites 1 and $N$ in our modified scheme. This quantity has been plotted in Fig 3 for various $M$ for large lengths of lattice up to $N=1001$. We find that for $M=1000$, entanglement nearly equal to that of 4 singlets is generated across a distance of 1001 lattice sites, and this is more than 70 times the amount possible with a spin- $1 / 2$ chain $(M=1)$ of same length. For high $N$ and $M$, we can represent $P_{r}$ by a Poisson distribution and know that $f_{1}(t) \sim 1.7 / N^{1 / 3}$ at $t \sim\left(N+0.81 N^{1 / 3}\right) / 4 J[2,11$. Using these, we have the analytic expression $\left\langle E_{v}\right\rangle \sim \log _{2}\left\{1.7 \sqrt{M \pi e} / N^{1 / 3}\right\}$, whose fit with data gets better as we proceed from the $M=10$ to the $M=1000$ plot at high $N$. For every order of magnitude increase of $M$ we thus expect to gain an entanglement equalling that of $\log _{2} \sqrt{10}=1.66$ singlets, which is confirmed by the differences in $\left\langle E_{v}\right\rangle$ between the $M=100$ and 1000 plots at high $N$ (Fig [3). So for obtaining entanglement exceeding that of 10 singlets across a distance of $\sim 1000$ lattice sites we require $M \sim 10^{7}$.

Now we proceed to compare the amount of entanglement between sites 1 and $N$ generated by the above schemes with that obtainable from the ground state of an ideal Bose gas in the same lattice (to check whether we have gained from dynamics). Ground state entanglement between distinct regions of a Bose-Einstein condensate in a single trap has already been investigated [13, 14]. In our lattice setting, the ground state of $H$ is simply $\left(\frac{1}{\sqrt{N}} \sum_{j=1}^{N} a_{j}^{\dagger}\right)^{M}|0\rangle / \sqrt{M !}$. It thus suffices to replace $f_{1}(t)$ in the expression of $P_{r}(t)$ with $1 / \sqrt{N}$ to change from the dynamical state to the ground state. $E_{n}$ between sites 1 and $N$ for the ground state is plotted using asterisks in Fig[2] as a function of $N$ for $M=50$. We find that the entanglement of sites 1 and $N$ is nearly vanishing for $N \geq 25$, and thus there is a marked advantage of using dynamics as opposed to the ground state. One may also however, measure the occupation numbers of sites 2 to $N$ of the ground state to obtain an average entanglement $\left\langle E_{v}\right\rangle \sim \log _{2} \sqrt{M \pi e / N}$ between sites 1 and $N$ (for high $M$ and $N)$. Even this is lower than that of our second scheme by $\log _{2}\left\{1.7 N^{1 / 6}\right\}$.

We now proceed to discuss a method to verify the pure entangled states $\left|\psi_{r}\right\rangle_{1 N}$ created through our second scheme. One can easily verify the number correlations (the fact that if site 1 has $k$ bosons, then the site $N$ has $r-k$ bosons) in $\left|\psi_{r}\right\rangle_{1 N}$ by occupation number measurements. Then the only task remaining is to verify the coherence between the terms $|k\rangle_{1}|r-k\rangle_{N}$ in $\left|\psi_{r}\right\rangle_{1 N}$. If we apply a Hamiltonian $H_{v}=\kappa a_{1}^{\dagger} a_{1}$ for a fixed time $t_{v}$ to site 1 then the above coherence leads to $\left|\psi_{r}\right\rangle_{1 N}$ becoming $\left|\psi_{r}^{\prime}\right\rangle_{1 N}=\left(e^{i \theta} a_{1}^{\dagger}+a_{N}^{\dagger}\right)^{r}|0\rangle / \sqrt{2^{r} r !}$ where $\theta=\kappa t_{v}$. The ideal Bose gas is then allowed to evolve again in the lattice (assuming the lattice sites 2 to $N-1$ have been emptied due to or after the earlier occupation number measurements), which results in its state becom$\operatorname{ing}\left|\Psi^{\prime}(t)\right\rangle_{1 . . N}=\left(\sum_{j=1}^{N}\left\{e^{i \theta} g_{1 j}(t)+g_{N j}(t)\right\} a_{j}^{\dagger}\right)^{r}|0\rangle / \sqrt{2^{r} r !}$

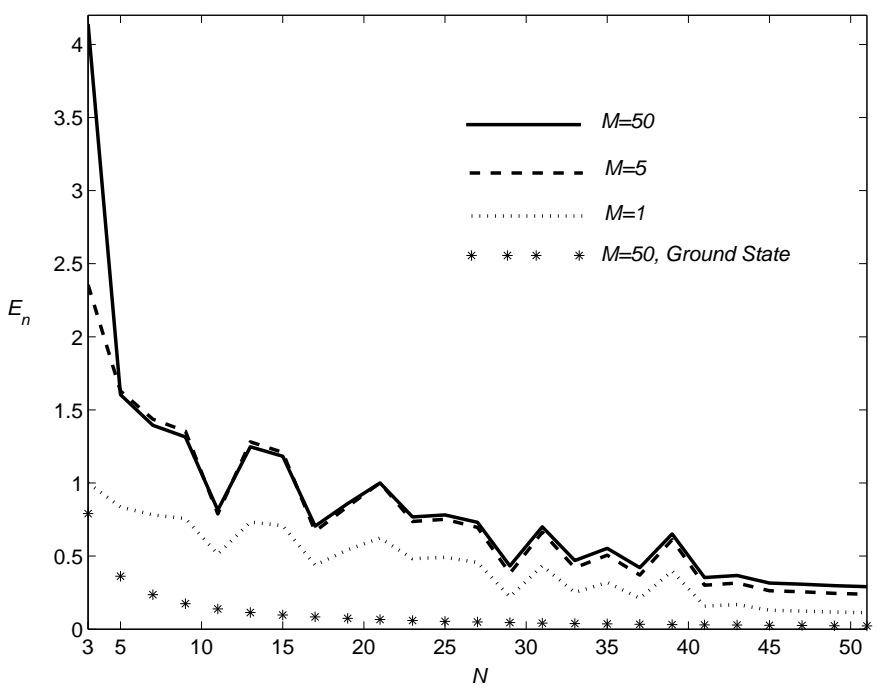

FIG. 2: Entanglement (log negativity) of sites 1 and $N$ from dynamics at an optimal time for various $N$ and $M$, and for the ground state.

where $g_{l j}$ are amplitudes for a boson going from the $l$ th site to the $j$ th site. From symmetry and the formulae for evolution in XY chain, we have $g_{1 \frac{N+1}{2}}(t)=g_{N \frac{N+1}{2}}(t)=$ $f_{1}(t)$. Thus the probability of finding the site $\frac{N+1}{2}$ occupied can be varied from $1-\left(1-2\left|f_{1}(t)\right|^{2}\right)^{r}$ for $\theta=0$, to 0 for $\theta=\pi$ (as the term $a_{\frac{N+1}{2}}^{\dagger}$ in $\left|\Psi^{\prime}(t)\right\rangle_{1 . . N}$ vanishes for $\theta=\pi)$. This variation with ${ }^{2} \theta$, whose range increases with increasing $r$ and which can be further increased by maximizing $\left|f_{1}(t)\right|$, enables us to verify the coherence between the terms $|k\rangle_{1}|r-k\rangle_{N}$ in $\left|\psi_{r}\right\rangle_{1 N}$. One way to verify the mixed state $\rho(t)_{1 N}$ produced by our first scheme will be to go through our second scheme and check that pure states $\left|\psi_{r}\right\rangle_{1 N}$ are produced with probabilities $P_{r}$.

Next, we provide an example of linking qubits $A$ and $B$ of distant quantum registers by making them interact with sites 1 and $N$ respectively for a time $t_{q}$, when these sites are in the state $\left|\psi_{r}\right\rangle_{1 N}$. The initial state of the qubits is $\left(|0\rangle_{A}+|1\rangle_{A}\right) \otimes\left(|0\rangle_{B}+|1\rangle_{B}\right) / 2$ and the interaction Hamiltonian is $H_{q}=g\left(\sigma_{z}^{A} a_{1}^{\dagger} a_{1}+\right.$ $\left.\sigma_{z}^{B} a_{N}^{\dagger} a_{N}\right)$ in which $\sigma_{z}^{A}$ and $\sigma_{z}^{B}$ are Pauli operators of the qubits. For $g t_{q}=\pi$, the state of the system is $\left\{\left(|0\rangle_{A}|0\rangle_{B}+(-1)^{r}|1\rangle_{A}|1\rangle_{B}\right) \otimes\left(a_{1}^{\dagger}+a_{N}^{\dagger}\right)^{r}|0\rangle+\left(|0\rangle_{A}|1\rangle_{B}+\right.\right.$ $\left.\left.(-1)^{r}|1\rangle_{A}|0\rangle_{B}\right) \otimes\left(a_{1}^{\dagger}-a_{N}^{\dagger}\right)^{r}|0\rangle\right\} / \sqrt{2^{r+2} r !}$. As in the previous paragraph, now the state of the ideal Bose gas is again allowed to evolve in the lattice and after some time the presence of any boson the $(N+1) / 2$ th site is measured. If this site is found occupied, then, remembering the logic of the last paragraph, the gas must have been in the state $\left(a_{1}^{\dagger}+a_{N}^{\dagger}\right)^{r}|0\rangle / \sqrt{2^{r} r}$, which projects the qubits to the maximally entangled state $|0\rangle_{A}|0\rangle_{B}+(-1)^{r}|1\rangle_{A}|1\rangle_{B}$. The probability for this is $1 / 2$. Otherwise (also with probability $1 / 2$ ), a state whose fi- 


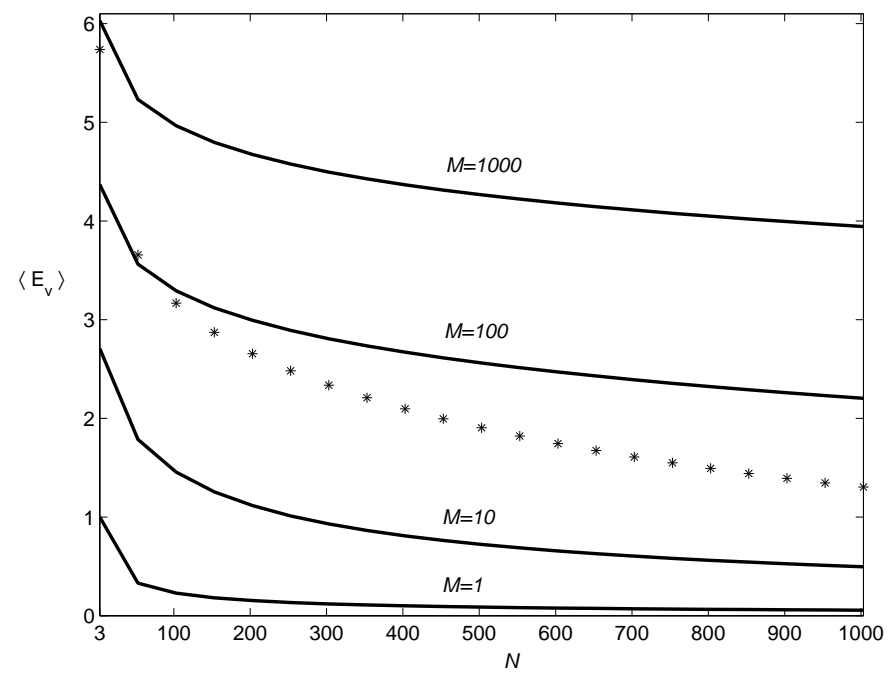

FIG. 3: Average entanglement (von Neumann entropy) of sites 1 and $N$ from dynamics followed by measurements on the intermediate lattice sites for various $N$ and $M$. The plot with asterisks denotes the same for the ground state for $M=1000$.

delity with $|0\rangle_{A}|1\rangle_{B}+(-1)^{r}|1\rangle_{A}|0\rangle_{B}$ is $1-\left(1-2\left|f_{1}(t)\right|^{2}\right)^{r}$ is obtained (this fidelity can be maximized by maximizing $\left|f_{1}(t)\right|$, and gets better with increasing $\left.r\right)$. This scheme for entangling qubits of distinct registers is just an example. The values of $\left\langle E_{v}\right\rangle$ found earlier imply that in principle one should be able to extract many more singlets from $\left|\psi_{r}\right\rangle_{1 N}$ by local actions alone.

BECs of dilute atomic gases in optical lattices form a test ground for our protocols. Fock states can be prepared by Mott transitions [15], the interactions needed for that can be switched off by a Feshbach resonance 16. to obtain an ideal Bose gas (otherwise, one simply has to go between regimes were the on-site repulsion $U>>J / M^{2}$ to $U<<J / M^{2} N$ by the global modulation of lattice potentials). Accurate measurement of the total number of bosons in sites 2 to $N-1$ is possible either by using metastable atoms [17] or potentially through a second Mott transition involving these sites only (note that individual site occupation numbers are not required). For the verification part, only whether the $(N+1) / 2$ th site is occupied or not need to be ascertained, and atomic fluorescence in external fields can potentially be used. By placing all atoms in a known magnetic state when required (say by a laser), each site can be imparted a magnetic moment proportional to its occupation number. This, and a local magnetic field at site 1 can be used to realize $H_{v}$. This magnetic moment also enables magnetic moment based register qubits (atomic or solid state) to interact with sites 1 and $N$ through $H_{q}$. If imparting the atoms with a magnetic moment automatically make $U>>J / M^{2}$ then we have to ensure that $U t_{v}$ and $U t_{q}$ are integral multiples of $2 \pi$. An alternative implementation is nano-oscillators arrays [5] when resonantly coupled to each other. They can be coupled to Cooper-pair box qubits to both create and measure Fock states [18], and to Josephson qubits through a Jaynes-Cummings model [19] whose off resonant limit can implement $H_{q}$. Coupled cavities in photonic crystals, where Fock state preparation and measurements could be performed with dopant atoms is another potential implementation [20].

I thank EPSRC for an Advanced Research Fellowship and for support through the grant GR/S62796/01 and the QIPIRC (GR/S82176/01).

[1] D. Kielpinski, C. Monroe and D. J. Wineland, Nature 417, 709 (2002)

[2] S. Bose, Phys. Rev. Lett 91, 207901(2003); D. Burgarth and S. Bose, Phys. Rev. A 71, 052315 (2005); V. Giovannetti and D. Burgarth, Phys. Rev. Lett. 96, 030501 (2006); T. Boness, S. Bose, and T. S. Monteiro, Phys. Rev. Lett. 96, 187201 (2006); J. Fitzsimons and J. Twamley, Phys. Rev. Lett. 97, 090502 (2006).

[3] M. Christandl, N. Datta, A. Ekert and A. J. Landahl, Phys. Rev. Lett. 92, 187902 (2004).

[4] M.-H. Yung and S. Bose, Phys. Rev. A 71, 032310 (2005).

[5] J. Eisert, M. B. Plenio, S. Bose and J. Hartley, Phys. Rev. Lett. 93, 190402 (2004); M.B. Plenio, J. Hartley and J. Eisert, New J. Phys. 6, 36 (2004).

[6] J. A. Dunningham and K. Burnett, Phys. Rev. A 61, 065601 (2000); J. A. Dunningham, K. Burnett and M. Edwards, Phys. Rev. A 64, 015601 (2001).

[7] J. A. Dunningham and K. Burnett, Phys. Rev. A 70, 033601 (2004).

[8] A. P. Hines, R. H. McKenzie and G. J. Milburn, Phys.Rev.A 67, 013609 (2003).

[9] B. Deb an G. S. Agarwal, Phys. Rev. A 67, 023603 (2003).

[10] J. A. Dunningham, K. Burnett, R. Roth and W. D. Phillips, quant-ph/0608242

[11] M.-H. Yung, D. W. Leung, and S. Bose, Quantum Inf. Comput. 4, 174 (2003).

[12] G. Vidal and R. F. Werner, Phys. Rev. A 65, 032314 (2002); M. B. Plenio, Phys. Rev. Lett. 95, 090503 (2005).

[13] C. Simon, Phys. Rev. A 66, 052323 (2002).

[14] L. Heaney, J. Anders and V. Vedral, quant-ph/0607069 V. Vedral and D. Kaszilikowski, quant-ph/0606238 D. Kaszilikowski et. al., quant-ph/0601089

[15] M. Greiner et. al., Nature 415, 39 (2002); C. Orzel et. al., Science 291, 2386 (2001).

[16] S. Inouye et. al. Nature 392, 151 (1998); J. L. Roberts et. al., Phys. Rev. Lett. 86, 4211 (2001).

[17] A. Robert et. al, Science 292, 461 (2001).

[18] E. K. Irish and K. Schwab, Phys. Rev. B 68, 155311 (2003).

[19] M. R. Geller and A. N. Cleland, Phys. Rev. A 71, 032311 (2005)

[20] D. G. Angelakis, M. F. Santos and S. Bose, quant-ph/0606159 M. J. Hartmann, F. G. S. L. Brandão and M. B. Plenio, quant-ph/0606097 A. D. Greentree et. al., cond-mat/0609050 